\title{
On the Transition from Bulk to Ordered Form of Water: A Theoretical Model to Calculate Adhesion Force Due to Capillary and van der Waals Interaction
}

\author{
M. A. Yaqoob • M. B. de Rooij • D. J. Schipper
}

Received: 24 August 2012/ Accepted: 21 December 2012/Published online: 11 January 2013

(C) The Author(s) 2013. This article is published with open access at Springerlink.com

\begin{abstract}
The adhesion force due to capillary interaction between two hydrophilic surfaces is strongly dependent on the partial pressure of water and is often calculated using the Kelvin equation. The validity of the Kelvin equation is questionable at low relative humidity $(\mathrm{RH})$ of water, like in high vacuum and dry nitrogen environments, where water is only present as layers of several molecules thick at the surfaces. A model from ordered to bulk form of water has been developed using the Brunauer, Emmett, and Teller adsorption model. The results show that the adhesion force calculated using the Young-Laplace and Kelvin equations at low (5-30 \%) RH is underestimated. The total adhesion force shows changes when the RH is changed from 0 to $100 \%$. In dry conditions, at RH below $10 \%$, the total adhesion force is contributed by the van der Waals interaction due to solid-solid contact. The total adhesion force then increases and remains constant being equal to the superposition of van der Waals interaction due to solidsolid contact and van der Waals interaction due to adsorbed water layers on the surfaces. The total adhesion force further increases slowly with the increase in RH incorporating capillary forces and then decreases at very high $\mathrm{RH}$ due to screening of van der Waals forces. This change in adhesion force occurs from solid-solid interaction to ordered form of water at low RH and from ordered form to bulk form of water at high $\mathrm{RH}$ along with the screening
\end{abstract}

M. A. Yaqoob

Materials Innovation Institute, Mekelweg 2, 2628 CD Delft, The Netherlands

M. A. Yaqoob $(\bowtie) \cdot$ M. B. de Rooij · D. J. Schipper Laboratory of Surface Technology and Tribology,

University of Twente, Drienerlolaan 5, 7522 NB Enschede,

The Netherlands

e-mail: m.a.yaqoob@ctw.utwente.nl; adeelyaqoob@yahoo.com effect of van der Waals interaction. The results have been compared with the experiments and it has been seen that at small length scales, the model is in agreement with the existing experimental data. However, at large length scales roughness of the surfaces should be taken into account.

Keywords Capillary interaction - van der Waals interaction - Adhesion force - BET adsorption model . Ordered water layers · Relative humidity · Film thickness

\section{Introduction}

An adhesion force is present between two surfaces when they are in contact with each other. The work of adhesion is the work need to separate these two surfaces and can be used to calculate the magnitude of the total adhesion force present in the system. However, the adhesion force is generally caused by the superposition of different kind of surface forces like van der Waals force, capillary force, electrostatic force, and other interacting forces present in a system as shown in Eq. (1) [1, 2].

$F_{\mathrm{a}}=F_{\text {cap }}+F_{\mathrm{vdw}}+F_{\text {el }}+\ldots$

where, $F_{\mathrm{a}}(\mathrm{N})$ is the adhesion force, $F_{\text {cap }}(\mathrm{N})$ is the capillary force, $F_{v d w}(N)$ is the van der Waals force, and $F_{e l}(N)$ is the electrostatic force.

Capillary forces or meniscus forces are present when the surfaces are in contact or are close to each other under humid conditions. This force strongly depends on relative humidity $(\mathrm{RH})$, roughness of the contacting bodies, radius of the sphere, and the hydrophilicity of the contacting bodies. The RH dependence of the capillary force has been reported for different kind of material combinations [2-5]. In other studies it was seen that due to a large surface 
roughness the adhesion due to capillary condensation is small compared to other surfaces with low surface roughness [6-8].

The validity of Kelvin's equation under these conditions is questionable. At $\mathrm{RH}=10 \%$ a Kelvin radius of $5.4 \AA$ is calculated which is approaching the size of a water molecule [6]. Therefore, the Kelvin's equation at very low RH $(\mathrm{RH}<10 \%)$ cannot be applied [6]. A similar argument was reported by Grobelny et al. [4] that the existing theories based on continuum mechanics are not sufficient for precise computation of capillary forces at very low RH values. Therefore, a model for calculating the adhesion force at low $\mathrm{RH}$ is required which can also incorporate the effect of adsorption layers on the surface.

Brunauer, Emmett, and Teller extended the Langmuir monolayer adsorption model to a more practical multilayer adsorption model. The model is called the BET adsorption model or BET adsorption isotherm [9]. The BET model can be used to estimate the film thickness on the surface as a function of the RH. There are some assumptions used in this model which limits its applicability in the whole range of RH; however, for the first estimate of the adsorbed film thickness this model is suitable. On the other hand, experiments have been performed to measure the adsorption layers on surfaces using attenuated total reflection infrared spectroscopy [10]. Verdaguer et al. [11] studied the growth and structure of water on $\mathrm{SiO}_{2}$ by several techniques, like surface potential measurements using an atomic force microscope (AFM) and ambient pressure $\mathrm{X}$-ray photoemission and near edge X-ray absorption fine structure spectroscopies (XPS and NEXAFS). The measurement results also followed the BET adsorption isotherm in a certain $\mathrm{RH}$ range which indicates the applicability of the model in that range.

In this paper a transition model for calculating the adhesion force as a function of partial pressure of water will be discussed. The model shows three transitions in the total adhesion force in the whole range of RH (0-100\%). At very low RH the dry situation is depicted and only solid-solid contact is formed. In this region only the van der Waals force contributes to the total adhesion force. At the intermediate $\mathrm{RH}$ region the van der Waals force due to adsorbed water layers will also contribute to the total adhesion force. In this region the water acts like a solid since the water molecules on the surface are in an ordered form. As the RH increases further the capillary force due to capillary formation of the bulk water will also contribute to the total adhesion force. At higher RH the total adhesion force decreases due to the screening of the van der Waals force. The results were compared with experimental data where experiments were performed in ambient conditions $\left(25{ }^{\circ} \mathrm{C}, 1\right.$ bar and $45 \% \mathrm{RH}$ ), ambient RH controlled conditions $\left(25{ }^{\circ} \mathrm{C}, 1\right.$ bar and $\left.10-45 \% \mathrm{RH}\right)$, and in high vacuum conditions $\left(25{ }^{\circ} \mathrm{C}, 10^{-6}\right.$ mbar $)$. Furthermore, results of the purposed model have been compared with the experimental results presented in literature.

\section{Theoretical Model}

The capillary force for the sphere-plane geometry, as shown in Fig. 1 can be calculated using Eq. (2). The capillary force is strongly influenced by the nature of surfaces in consideration. Since the attractive capillary force is given as [2, 8]:

$F_{\text {cap }}=-2 \pi R \gamma_{\mathrm{L}}\left(\cos \theta_{1}+\cos \theta_{2}\right)$

where, $R(m)$ is the radius of the sphere, $\theta_{1,2}$ are the contact angles between the liquid and the surfaces, and $\gamma_{L}$ is the surface tension of the liquid (here water).

The capillary force is directly influenced by the contact angles of the surfaces. Similarly, from Eq. (2) it can also be seen that there is no RH dependence on the capillary force. However, there is strong experimental evidence of $\mathrm{RH}$ dependence of the capillary force [2-5]. There are several reasons which restricts the applicability of Eq. (2) like the filling angle $\varphi \approx 0$ if the $R \gg r_{2} \gg r_{1}$ [2, 12]. First, the filling angle is dependent on the RH where the meniscus radius $r_{2}$ increases with an increase in $\mathrm{RH}$. As the amount of water in the system increases the meniscus will grow and will increase the meniscus radius $r_{2}$. Second, for a small tip radius $R$ e.g., an AFM tip the $R \gg r_{2} \gg r_{1}$ approximation fails [2]. Third, there is no contribution from the surface tension force in the total capillary force shown in Eq. (2), which is again an important contribution for small radii tips.

The water is considered in structured form or ordered form until three monolayers of water molecules and then there is a transition from ordered to bulk [10]. Therefore,

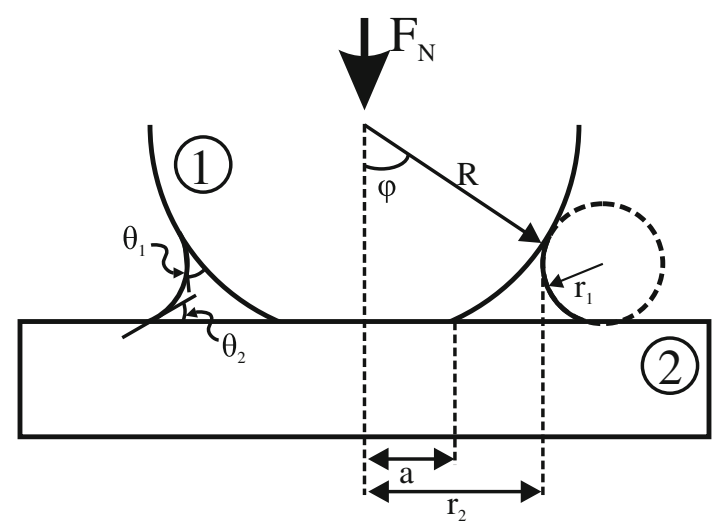

Fig. 1 A sphere in contact with a flat surface under a certain applied normal load $F_{\mathrm{N}}$ in humid environment. The solid-solid contact radius $a$ and the meniscus radius $r_{2}$ are also shown 
the Kelvin equation cannot be used to calculate the adhesion force for low RH. The force acting on the sphere due to meniscus formation when the meniscus is in equilibrium is written as [3]:

$$
\begin{aligned}
& F_{\mathrm{c}}=F_{\mathrm{s}}+F_{\mathrm{p}} \\
& F_{\mathrm{s}}=-2 \pi \gamma_{\mathrm{L}} r_{2} \sin \left(\theta_{1}+\varphi\right)=2 \pi \gamma_{\mathrm{L}} R \sin \varphi \sin \left(\theta_{1}+\varphi\right)
\end{aligned}
$$

$$
\begin{aligned}
F_{\mathrm{p}} & =-\Delta p \pi\left(r_{2}^{2}-a^{2}\right) \\
& =-\left[-\frac{R_{\mathrm{g}} T}{V_{\mathrm{m}}} \log \left(\frac{p}{p_{\mathrm{s}}}\right)\right] \pi\left(R^{2} \sin ^{2} \varphi-a^{2}\right)
\end{aligned}
$$

where, $F_{\mathrm{s}}$ is the surface tension force and $F_{\mathrm{p}}$ is the capillary pressure force which is attractive because the pressure in the liquid is lower than in the outer vapor phase. The negative sign shown in Eq. (5) is due to the fact that the log $\left(p / p_{\mathrm{s}}\right)<0$. In Eq. (5) the capillary pressure $\Delta p$ is given using the Young-Laplace and Kelvin equations. $R_{\mathrm{g}}$ is the universal gas constant, $T$ is the absolute temperature, $V_{\mathrm{m}}$ is the molar volume, $p$ and $p_{\mathrm{s}}$ are the equilibrium and saturation vapor pressures of the liquid, respectively, and $a$ is the contact radius for solid-solid contact which can be calculated by e.g., Hertz theory [13].

It can be seen that the total capillary force $F_{\mathrm{c}}$ can be calculated if the filling angle $\varphi$ is known. This filling angle can be calculated implicitly using the Young-Laplace and Kelvin equations along with the geometrical analysis of the contact situation shown in Fig. 1 and is given as [2]:

$\frac{\Delta p}{\gamma_{\mathrm{L}}}=\left(\frac{1}{r_{1}}+\frac{1}{r_{2}}\right)=-\frac{R_{\mathrm{g}} T}{V_{\mathrm{m}} \gamma_{\mathrm{L}}} \log \left(\frac{p}{p_{\mathrm{s}}}\right)$

$-\frac{R_{\mathrm{g}} T}{V_{\mathrm{m}} \gamma_{\mathrm{L}}} \log \left(\frac{p}{p_{\mathrm{s}}}\right)=\frac{\cos \left(\theta_{1}+\varphi\right)+\cos \theta_{2}}{d+R(1-\cos \varphi)}+\frac{1}{R \sin \varphi}$

Here $d$ is the distance between the sphere and the flat (not shown in Fig. 1) which is assumed to be $0.2 \mathrm{~nm}$ when the sphere and flat are in contact [1]. It can be seen that by simplifying Eq. (5) using the assumptions explained above, Eq. (2) can be obtained.

In the solid-solid contact, without the meniscus, the van der Waals force will only contribute to the total adhesion force. This solid-solid contact situation can also be termed as the dry contact situation when there will be very little water present (very low RH) in the environment like in high vacuum (HV) or ultra high vacuum (UHV). The van der Waals force for sphere-flat geometry can be calculated by [1]:

$F_{\mathrm{S}-\mathrm{vdw}}=-\frac{A_{132} R}{6 d^{2}}$

where, $A_{132}$ is the Hamaker constant between sphere 1 and flat 2 in medium 3 (here vacuum). As mentioned earlier the distance $d$ is assumed to be $0.2 \mathrm{~nm}$ when the surfaces are in contact; therefore, the van der Waals force due to solidsolid contact is a constant for a certain set of materials of constant geometry.

As the amount of water content in the environment increases the adsorption of water molecules on the surfaces will also increase. The thickness of this adsorbed water layer can be calculated using BET adsorption isotherm [9]. The film thickness $T_{f}$ as a function of RH is given as [9]:

$$
\begin{aligned}
T_{\mathrm{f}}= & \frac{C \cdot n_{\mathrm{mon}} \cdot m \cdot \sigma_{\mathrm{A}}}{\left(1-\frac{p}{p_{\mathrm{s}}}\right) \cdot\left[1+\left\{\frac{p}{p_{\mathrm{s}}} \cdot(C-1)\right\}\right]} \cdot \frac{p}{p_{\mathrm{s}}} \\
& \because C=e^{\left(\mathrm{Q}_{1}-\mathrm{Q}_{\mathrm{i}}\right) / \mathrm{RT}}
\end{aligned}
$$

where, $C$ is called the BET constant which is dependent on the $Q_{1}$ and $Q_{\mathrm{i}}$ which are the heat of adsorption of the first layer and heat of condensation of the adsorbate (here water), respectively. The $\mathrm{n}_{\text {mon }}$ is the number of adsorbed molecules in one full monolayer per unit area, $\sigma_{\mathrm{A}}$ is the cross-sectional area, and $m$ is the diameter of the adsorbate molecule (for water, $\sigma_{\mathrm{A}}$ is $0.77 \mathrm{~nm}^{2}$ and $m$ is approximately $2.75 \AA$ ) $[14,15]$. The typical values of $C$ and $\mathrm{n}_{\text {mon }}$ for different materials have been measured and it was shown that the film thickness is strongly dependent on the hydrophilicity of the adsorbent [16]. The $C$ values used further for non-porous $\alpha$-alumina and silica are 28 and 11 and the corresponding $\mathrm{n}_{\text {mon }}$ values are 6.5 and 3.6 molecules per $\mathrm{nm}^{2}$, respectively $[9,16]$.

The BET isotherm for silica is shown in Fig. 2 and is compared with the values of measured film thickness from literature [10]. It can be seen that the measured values fit well until $50 \% \mathrm{RH}$ which is a strong evidence to use BET isotherm for calculating film thickness until $50 \% \mathrm{RH}$ for silica. The first monolayer forms at about $10 \% \mathrm{RH}$; therefore, we can consider solid-solid contact before $10 \%$ RH. After $10 \% \mathrm{RH}$ the monolayer starts to grow in multilayers and at about $30 \% \mathrm{RH} 3$ monolayers are formed. The water will be considered as bulk only if the number of monolayers is greater than $3[10,11]$. So, water will be in an ordered form from 10 to $30 \% \mathrm{RH}$. The film thickness keeps on growing slowly and there is a transition from ordered to bulk form of water between 30 and $60 \% \mathrm{RH}$. After $60 \% \mathrm{RH}$ the meniscus will be formed around the sphere-flat contact and the water layers can be considered as bulk. However, the increase in the amount of water in the contact will decrease the van der Waals force due to more adsorbed layers of water. This is called the screening of van der Waals force [1].

The contact situations representing three different regions of RH are schematically shown in Fig. 3. The van der Waals force between 10 and $30 \% \mathrm{RH}$ considering the 


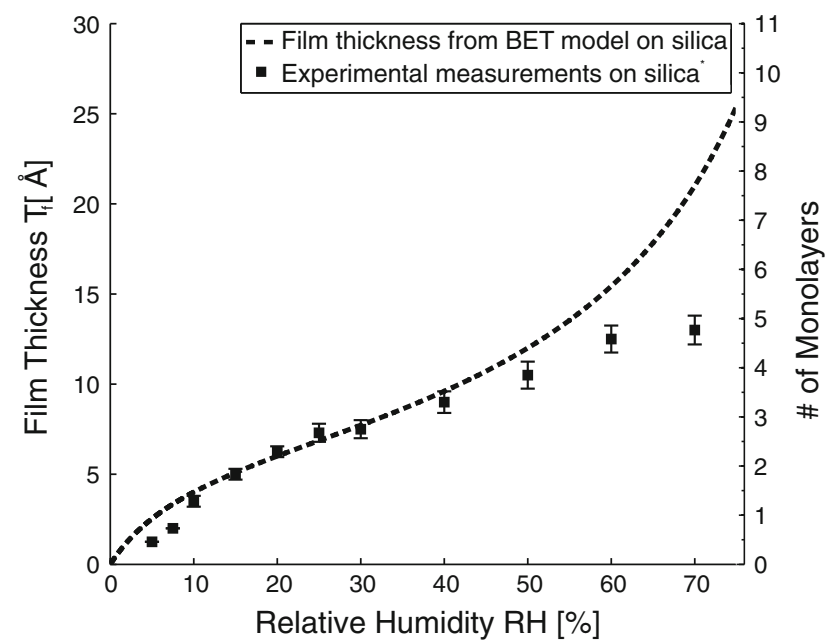

Fig. 2 Water film thickness as a function of RH on silica surface calculated using BET adsorption isotherm. The theoretical values have been compared with the measurement results from literature [10]

adsorbed layer system between a sphere and a flat surface $F_{\mathrm{W}-\mathrm{vdw}}$ as shown in Fig. $3 \mathrm{~b}$ is given by [1]:

$F_{\mathrm{W}-\mathrm{vdw}}$

$=-\frac{R}{6}\left[\frac{A_{\mathrm{waw}}}{d^{2}}-\frac{\sqrt{A_{1 \mathrm{w} 1} A_{\mathrm{awa}}}}{\left(d+T_{\mathrm{f} 1}\right)^{2}}-\frac{\sqrt{A_{2 \mathrm{w} 2} A_{\mathrm{awa}}}}{\left(d+T_{\mathrm{f} 1}\right)^{2}}+\frac{\sqrt{A_{2 \mathrm{w} 2} A_{1 \mathrm{w} 1}}}{\left(d+T_{\mathrm{f} 1}+T_{\mathrm{f} 2}\right)^{2}}\right]$

where, $T_{\mathrm{f} 1}$ is the water film thickness on the sphere $1, T_{\mathrm{f} 2}$ is the water film thickness on flat $2, w$ represents water, and $a$ represents air or vacuum.

It is important to mention here that the Hamaker constant $A_{\text {waw }}$ or $A_{\text {awa }}$ has been calculated using pairwise additivity rather than using Lifshitz theory. This is because the water molecules in 10-30 \% RH cannot be considered as bulk and the Lifshitz theory considers materials to be in bulk form and uses bulk properties. However, the transition region for other materials can be different as explained above depending on the trend of film thickness as a function of RH.

\section{Results and Discussion}

The above theory can be used to calculate the total adhesion force for a wide range of $\mathrm{RH}$. Considering the above analysis, Eq. (1) can be rewritten as:

$$
\begin{aligned}
& F_{\mathrm{a}}= \\
& \qquad \begin{array}{ll}
F_{\mathrm{S}-\mathrm{vdw}} & T_{\mathrm{f}} \leq 1 \text { monolayer } \\
F_{\mathrm{S}-\mathrm{vdw}}+F_{\mathrm{W}-\mathrm{vdw}} & 1 \text { monolayer }<T_{\mathrm{f}} \leq 3 \text { monolayers } \\
F_{\mathrm{S}-\mathrm{vdw}}+F_{\mathrm{W}-\mathrm{vdw}}+F_{\mathrm{c}} & T_{\mathrm{f}} \geq 3 \text { monolayers }
\end{array}
\end{aligned}
$$

Using Fig. 2 we can correlate the number of monolayers with the RH. It can be seen that there are two transitions in
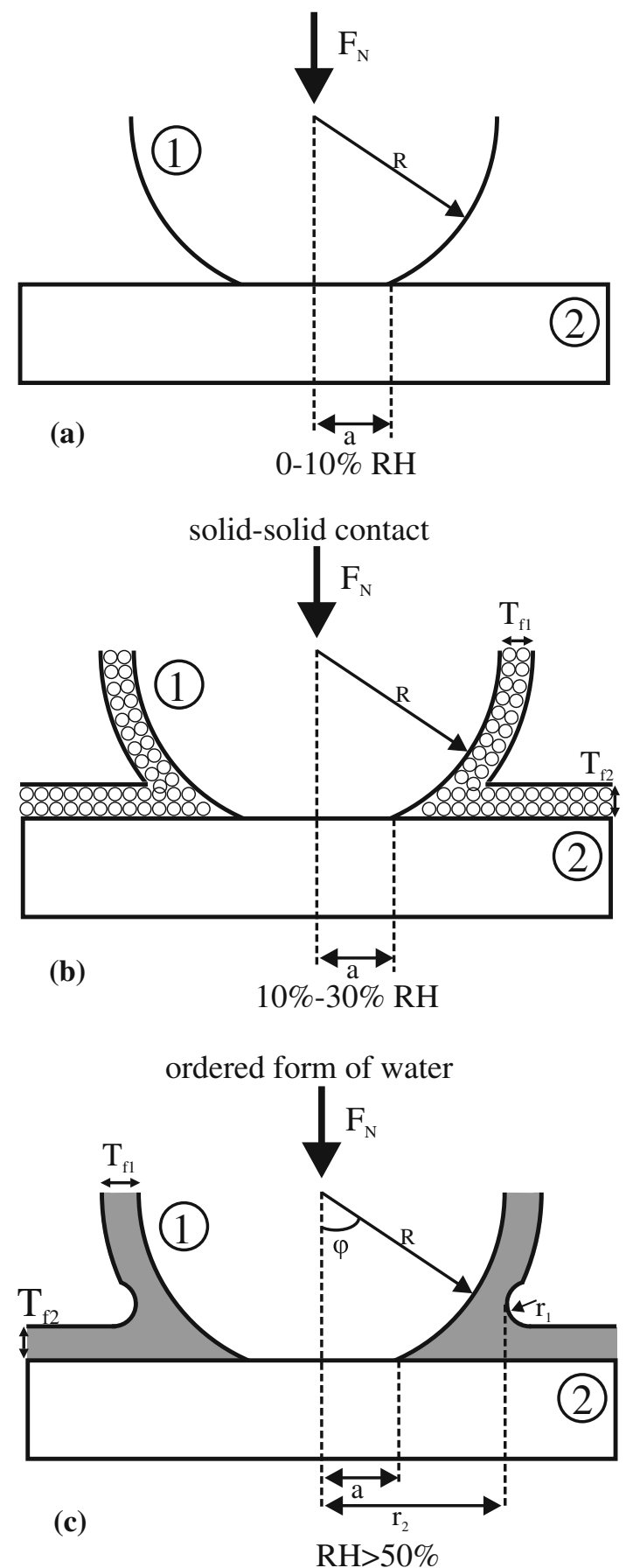

meniscus formation

Fig. 3 Schematic representation of different contact situations for different regions of RH. The solid-solid contact depicts the completely dry situation, the ordered form of water shows the monolayer formation due to adsorption, and meniscus formation shows a bulk water forming meniscus

the adhesion force as shown in Eq. (11). The first transition is from solid-solid to solid-solid + adsorbed layered system and the second one is from solid-solid + adsorbed layered to solid-solid + adsorbed layered + meniscus. 
S-shaped function has been used in both the transitions since it is physically realistic. It is important to mention here that the emphasis is on the adhesion force levels (e.g., $F_{\mathrm{S} \text {-vdw }}, F_{\mathrm{S} \text {-vdw }}+F_{\mathrm{W} \text {-vdw }}$, etc.) rather than the shape of the transitions. From Fig. 2 the first monolayer on silica is formed at approx. $10 \% \mathrm{RH}$ and three monolayers are formed at about $30 \%$ RH. For the first transition, for example, this $\mathrm{S}$-shaped curve in terms of $\mathrm{RH}$ could be given as:
This size of the contact geometry is used to compare with the results from literature. Three distinctive regions are shown in the Fig. 4 which depends on the RH of water and will be explained step by step as follows:

- At very low $\mathrm{RH}<1 \%$ there is no water since the adsorption phenomenon is very slow, as can be seen from Fig. 2. At this stage the adhesive force is contributed by the van der Waals force between the two solid surfaces and, therefore, called solid-solid contact.

$$
F_{\text {atran } 1}= \begin{cases}F_{\mathrm{S}-\mathrm{vdw}} & x \leq R H_{0} \\ F_{\mathrm{S}-\mathrm{vdw}}+\left[\left(F_{\mathrm{W}-\mathrm{vdw}}\right) \cdot\left(2 \cdot\left(\frac{x-R H_{0}}{R H_{1}-R H_{0}}\right)^{2}\right)\right] & R H_{0} \leq x \leq \frac{R H_{0}+R H_{1}}{2} \\ F_{\mathrm{S}-\mathrm{vdw}}+F_{\mathrm{W}-\mathrm{vdw}}-\left[\left(F_{\mathrm{W}-\mathrm{vdw}}\right) \cdot\left(2 \cdot\left(\frac{R H_{1}-x}{R H_{1}-R H_{0}}\right)^{2}\right)\right] & \frac{R H_{0}+R H_{1}}{2} \leq x \leq R H_{1} \\ F_{\mathrm{S}-\mathrm{vdw}}+F_{\mathrm{W}-\mathrm{vdw}} & x \geq R H_{1}\end{cases}
$$

where, $F_{\text {atran } 1}$ is the adhesion force in the transition region $R H_{0}-R H_{1}, R H_{0}$ is the start of the transition from dry situation to the first monolayer situation, $R H_{1}$ is the relative humidity where one complete monolayer is formed, and $x$ is the transition point between $R H_{0}$ and $R H_{1}$. Similar expressions have been used for the $\mathrm{RH}_{2}-\mathrm{RH}_{3}$ and $\mathrm{RH}_{4}-\mathrm{RH}_{5}$ transition regions shown in Fig. 4.

The results from the model for a silicon ball of $20 \mathrm{~nm}$ with a native oxide and a glass surface are shown in Fig. 4.

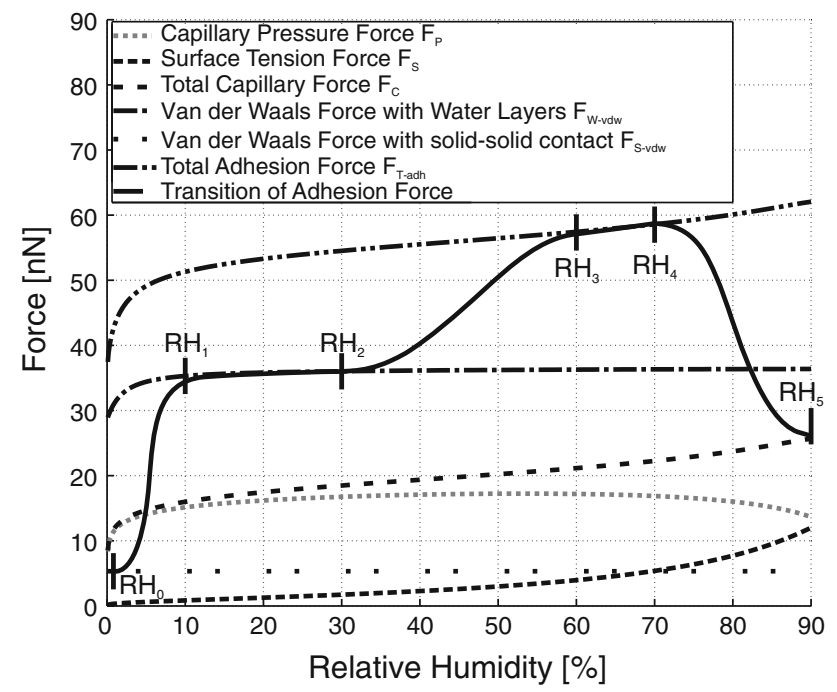

Fig. 4 Simulation results of the trends of different surface forces as a function of $\mathrm{RH}$ for a 20-nm-radius $\mathrm{SiO}_{2}$ ball and a glass flat. The solid-solid contact $F_{\mathrm{S} \text {-vdw }}$ depicts completely dry situation, the ordered form of water $F_{\mathrm{W} \text {-vdw }}$ shows the monolayer formation due to adsorption, and meniscus formation $F_{\mathrm{c}}$ shows bulk water forming meniscus. The transition line shows the transition in adhesion force at different $\mathrm{RH}$
- In the region of $1 \%<\mathrm{RH}>10 \%$ (between $\mathrm{RH}_{0}$ and $\mathrm{RH}_{1}$ in Fig. 4) the water molecules start to adsorb more rapidly and at $10 \% \mathrm{RH}$ the first monolayer is formed. In this region the transition is shown from solid-solid to an ordered layered system. The emphasis here is on the change in the level of adhesion force rather than the nature of the transition.

- From 10 to $30 \% \mathrm{RH}$ (between $\mathrm{RH}_{1}$ and $\mathrm{RH}_{2}$ in Fig. 4) there will be an ordering effect where the adhesive force is contributed by the van der Waals forces of adsorbed water layers on the surfaces along with the solid-solid van der Waals interaction.

- When $\mathrm{RH}>30 \%$ (between $\mathrm{RH}_{2}$ and $\mathrm{RH}_{3}$ in Fig. 4) a slow transition will start from ordered to bulk and at $60 \%$ the transition completes as the fourth monolayer is formed. At this stage the meniscus will be formed and the total adhesive force is contributed by the superposition of van der Waals force due to solid-solid contact, van der Waals force due to adsorbed water layers, and capillary force which can be calculated using Eq. (3). It can also be seen that at nano scale the surface tension force $F_{\mathrm{s}}$ cannot be neglected.

- As the RH increases the capillary forces dominate and the screening of van der Waals force can be seen above $70 \%$ (between $\mathrm{RH}_{4}$ and $\mathrm{RH}_{5}$ in Fig. 4). The adhesion force is reduced to the capillary force only at very high humidity.

It is important to mention here that these simulations have been performed by considering the contact between two smooth surfaces and there are no transient effects on the capillary formation. It can be seen that the total capillary force $F_{\mathrm{c}}$ calculated with Eq. (3) using the Kelvin 


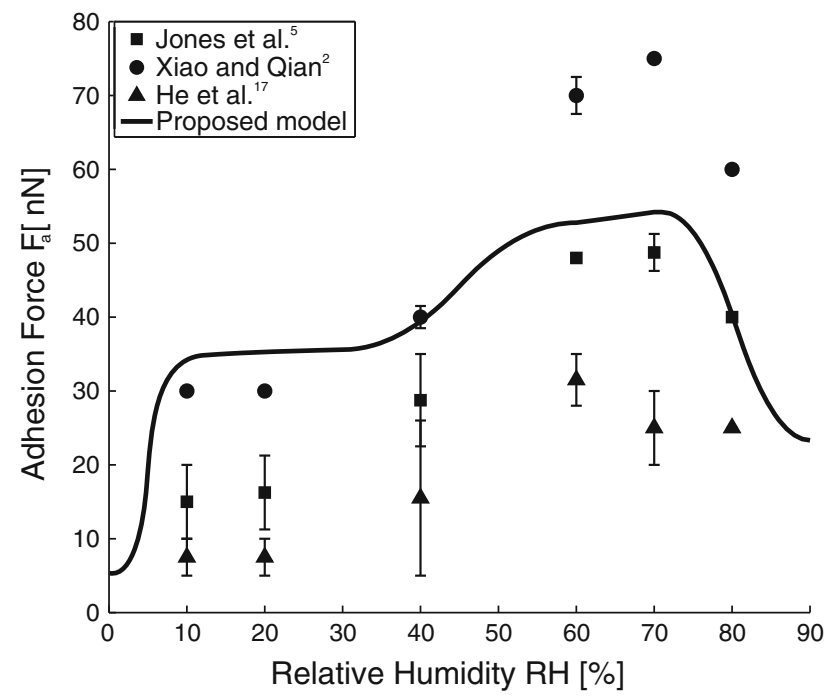

Fig. 5 Comparison of the adhesion force measured by Jones et al. [5], Xiao and Qian [2], He et al. [17], and calculated by the proposed model

equation underestimates the total adhesion force and does not predict the transition regions in the total adhesion force. The total adhesion force $\mathrm{F}_{\mathrm{T} \text {-adh }}$ is the superposition of all the adhesive forces present in the system. The transition curve (solid line) shows the transition between three distinct regimes as shown in Fig. 3. AFM pull-off measurements for different $\mathrm{RH}$ between $\mathrm{SiO}_{2}$ and $100 \mathrm{~nm}$ radius $\mathrm{Si}_{3} \mathrm{~N}_{4}$ tip had been reported by Xiao and Qian [2]. Similar results had been shown by Jones et al. [5] between 20-nmradius $\mathrm{SiO}_{2}$ ball and using glass and $\mathrm{SiO}_{2}$ as counter surfaces and by $\mathrm{He}$ et al. [17] between $\mathrm{Si}$ and 20-nm-radius $\mathrm{Si}_{3} \mathrm{~N}_{4}$ tips. Furthermore, the results from the selected literature shown in Fig. 5 do not show pull-off measurements at $\mathrm{RH}<10 \%$. The results from literature have been summarized in Fig. 5 and they show good agreement with the proposed model. The experimental data from the different literature sources is not quantitatively in mutual agreement as shown in Fig. 5. There are many possible reasons for these differences since the measurements have been performed with different experimental setups. However, from Fig. 5 it is clear that our model predicts the same order of magnitude of all these measurements.

Similar simulations have been carried out for a $\mathrm{SiO}_{2}$ ball of $5 \mathrm{~mm}$ diameter in contact with a glass flat surface. The calculated adhesion forces with the model are shown in Fig. 6. It can be seen that the contribution of the surface tension force $F_{\mathrm{s}}$ to the total capillary force $F_{\mathrm{c}}$ can be neglected for this radius. Therefore, the total capillary force is equal to the capillary pressure force $F_{\mathrm{p}}$. These simulations have been carried out to compare the results to the adhesion measurements performed on a dedicated Vacuum

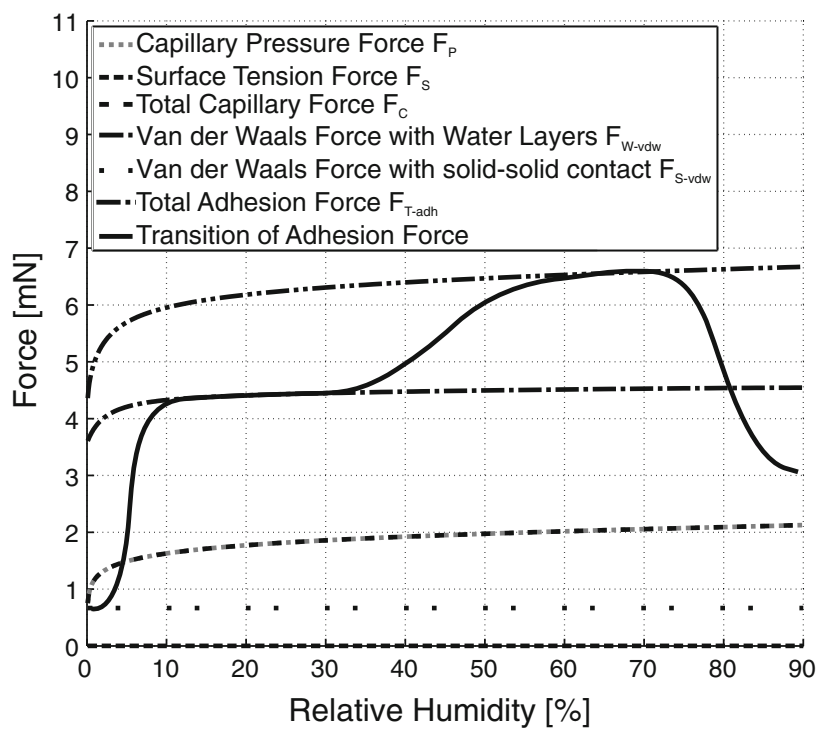

Fig. 6 Simulation results of the trends of different surface forces as a function of $\mathrm{RH}$ for a 5-mm-diameter $\mathrm{SiO}_{2}$ ball against a glass flat

Adhesion and Friction Tester (VAFT) shown in Fig. 7. The detailed design and working of the VAFT has been explained elsewhere [18].

\subsection{Experiments}

To validate the model the adhesion experiments were performed in ambient as well as in high vacuum conditions. The samples used in these experiments are 5-mmdiameter $\mathrm{SiO}_{2}$ ball and a borosilicate glass (float glass) flat surface. The samples are prepared in the following way before experiments.

\subsubsection{Sample Preparation}

A flat glass surface has been cleaned in an ultrasonic bath of acetone for $15 \mathrm{~min}$, rinsed with deionized water, and then dried. The silica ball after cleaning in an ultrasonic bath of acetone for $15 \mathrm{~min}$, rinsing in deionized water, and drying has been glued to an indenter. The ball has been inspected for any pits and bumps on the surface with the Keyence Confocal Microscope before applying the glue and the surface roughness was measured. The ball was also inspected after the glue was dried and no contamination due to the application of glue on surface was found. The surface roughness was measured after this step as well. To make sure that the surface is cleaned the glued ball along with the indenter was cleaned in an ultrasonic bath of acetone for $15 \mathrm{~min}$, rinsed with deionized water, and then dried. The glue used in this process was not reactive with acetone. The ball was again inspected using the standard 

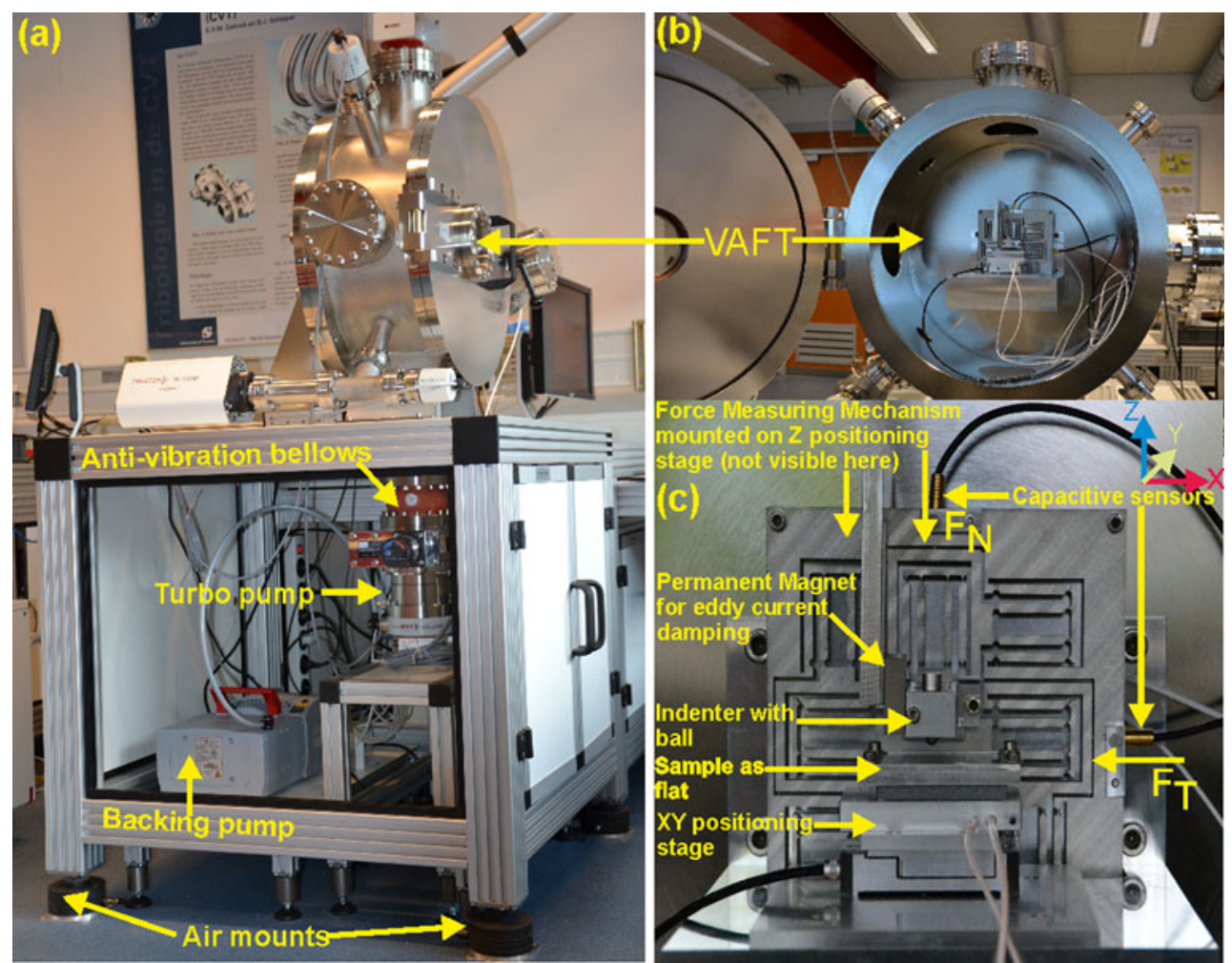

Fig. 7 Test setup for measuring adhesion and friction force in ambient and high vacuum conditions

inspection procedure and the surface roughness was measured. Both measurements showed that there is no contamination on the surface of the ball after glue. The flat glass has been placed on an XY positioning stage and the indenter is then mounted on the cantilever. The cantilever was then mounted through the force measuring mechanism on the $Z$ positioning stage, see Fig. 7. The whole setup has been placed in a vacuum chamber to control the RH. The

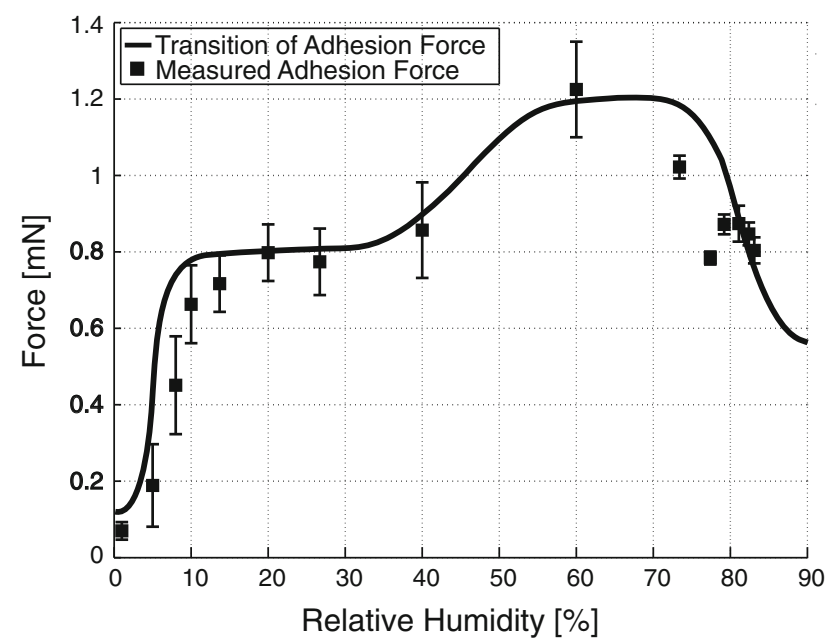

Fig. 8 Simulation results for adhesion force along with the measured adhesion force for a 5-mm-diameter $\mathrm{SiO}_{2}$ ball against a glass flat
$\mathrm{RH}$ is measured through a humidity sensor in the chamber and the RH can be controlled by flushing liquid nitrogen through a coil which traps the water molecules present in the chamber.

\subsubsection{Validation of the Model}

The measurement results for a 5-mm-diameter silica ball and glass interface along with the calculated results are shown in Fig. 8. The measurements have been performed in ambient conditions before and after pumping the chamber down to $10^{-7}$ mbar for several days. The measured adhesion force during this process has been well within the error margin indicated in Fig. 8. Furthermore, each measurement point consists of at least ten adhesion measurements.

The measurement points shown in Fig. 8 at very low relative humidity and high relative humidity has a small error bandwidth as compared to the measurements between 5 and $60 \% \mathrm{RH}$. The reason for this large bandwidth in this range could be because of the distribution of water layers on the surfaces. However, the measurement points with small bandwidth shows a dry contact at very low RH (the first point in Fig. 8) and the wet contact with bulk water effects at humidity between 70 and $80 \%$. The first point in Fig. 8 depicting the dry situation has been measured when the system has been pumped down to the pressure of $10^{-7}$ mbar. The pressure is then increased to $10^{-5} \mathrm{mbar}$ and it can be seen 
that the adhesion force is increased with respect to the measurement performed at $10^{-7}$ mbar. The increase in pressure increases the RH in the chamber and the adhesion force increases as the pressure is increased until 1 bar showing the formation of monolayers on the surfaces.

The measurement results as well as model results show the same trend; however, the magnitude of the adhesion force measured, as shown in Fig. 8 is approximately 5 times less than the magnitude calculated with the model as shown in Fig. 6. The main reason for this is that the model does not incorporate the roughness effects of the surfaces. Although the surface roughness values of $\mathrm{SiO}_{2}$-glass system are very small but the adhesion force is very sensitive to surface roughness $[7,8,19,20]$. An adhesion parameter $\theta$ was introduced which is a function of elastic modulus $E$, surface energy $\Delta \gamma$, asperity radius $\beta$, and standard deviation of the surface roughness $\sigma$ and is given by [19]:

$\theta=\frac{E \sigma^{\frac{3}{2}}}{\beta^{\frac{1}{2}} \Delta \gamma}$

If the value of the adhesion parameter is larger than 10, the adhesion force significantly reduces from the adhesion force value measured between relatively smooth surfaces [19]. It has also been reported that the adhesion force reduces two orders of magnitude with one order of magnitude change in rms roughness (i.e., $1-10 \mathrm{~nm}$ ) [8]. Similarly, value of adhesion force as a function of rms roughness has been reported to be decreasing by a factor of five if the rms roughness is increased from 0.2 to $4 \mathrm{~nm}$ and for higher roughness values it stabilizes [7, 21]. For the $\mathrm{SiO}_{2}$-glass system the value of adhesion parameter reaches ten when the rms roughness is $1 \mathrm{~nm}$. The measured rms roughness of $\mathrm{SiO}_{2}$ ball is $2-3 \mathrm{~nm}$ and the flat glass surface is $0.7-1 \mathrm{~nm}$. Therefore, equivalent roughness of the contact is higher than $1 \mathrm{~nm}$. There are small microcontacts present in the contact and, therefore, the real area of contact is much smaller than the nominal area of contact which reduces the pull-off force significantly. Furthermore, capillary force and the van der Waals force scales with the radius of the spherical surface (here contacting asperities) [1]. As mentioned earlier, the measured adhesion force is a factor five less than the calculated adhesion force from the purposed model and this difference corresponds to the study reported by van Zwol et al. [8] and Ata et al. [21] where the relation between the rms roughness and the adhesion force has been established.

\section{Conclusions}

A transition model for calculating adhesion force as a function of RH has been developed using BET adsorption isotherm. The results of the calculated adhesion force shows that the Kelvin equation cannot be used below a certain RH. The results also show that the Kelvin equation cannot predict the transition behavior of the adhesion force with changing RH. The model shows good agreement with the measurement results for a selected pair of materials from literature for nano scale contacts. However, at macro scale the roughness of the interface influences the adhesion force significantly. The calculated adhesion values from the model at macro scale have been compared with the measurements performed on a dedicated measurement setup. The trend of the effect of humidity on the adhesion force is well predicted. However, the measured adhesion force is 5 times less than the calculated adhesion force for macro scale which is caused by the roughness of the interface. This fact is also in agreement with literature.

Acknowledgments This research was carried out under project number MC7.06284 in the framework of the Research Program of the Materials innovation institute M2i (www.m2i.nl). Financial support for carrying out this research from the M2i is gratefully acknowledged.

Open Access This article is distributed under the terms of the Creative Commons Attribution License which permits any use, distribution, and reproduction in any medium, provided the original author(s) and the source are credited.

\section{References}

1. Israelachivili, J.N.: Intermolecular and Surface Forces, 2nd edn. Elsevier, London (1991)

2. Xiao, X., Qian, L.: Investigation of humidity-dependent capillary force. Langmuir 16, 8153-8158 (2000)

3. Butt, H.-J., Kappl, M.: Normal capillary forces. Adv. Colloid Interface 146, 48-60 (2009)

4. Grobelny, J., Pradeep, N., Kim, D.I., Ying, Z.C.: Quantification of the meniscus effect in adhesion force measurement. Appl. Phys. Lett. 88(091906), 1-3 (2006)

5. Jones, R., Pollock, H.M., Cleaver, J.A.S., Hodges, C.S.: Adhesion force between glass and silicon surfaces in air studied by AFM: effects of relative humidity, particle size, roughness and surface treatment. Langmuir 18, 8045-8055 (2002)

6. Feiler, A.A., Stiernstedt, J., Theander, K., Jenkins, P., Rutland, M.: Effect of capillary condensation on friction force and adhesion. Langmuir 23, 517-522 (2007)

7. Rabinovich, Y.I., Alder, J.J., Esayanur, M.S., Ata, A., Singh, R.K., Moudgil, B.M.: Capillary forces between surfaces with nanoscale roughness. Adv. Colloid Interface 96, 213-230 (2002)

8. van Zwol, P.J., Palasantzas, G., de Hosson, J.Th.M.: Influence of roughness on capillary forces between hydrophilic surfaces. Phys. Rev. E 78(031606), 1-6 (2008)

9. Butt, H.-J., Graf, K., Kappl, M.: Physics and Chemistry of Interfaces. Wiley-VCH, Weinheim (2003)

10. Asay, D.B., Kim, S.H.: Evolution of the adsorbed water layer structure on silicon oxide at room temperature. J. Phys. Chem. B 109(35), 16760-16763 (2005)

11. Verdaguer, A., Weis, C., Oncins, G., Ketteler, G., Bluhm, H., Slameron, M.: Growth and structure of water on $\mathrm{SiO}_{2}$ films on $\mathrm{Si}$ investigated by Kelvin probe microscopy and in situ X-ray spectroscopies. Langmuir 23, 9699-9703 (2007) 
12. Xu, D., Liechti, K.M., Ravi-Chandar, K.: On the modified tabor parameter for the JKR-DMT transition in the presence of a liquid meniscus. J. Colloid Interface Sci. 315, 772-785 (2007)

13. Johnson, K.L.: Contact Mechanics. Cambridge University Press, Cambridge (1985)

14. Mizushima, S.: Determination of the amount of gas adsorption on $\mathrm{SiO}_{2} / \mathrm{Si}(100)$ surfaces to realize precise mass measurement. Metrologia 41, 137-144 (2004)

15. Mizushima, S.: The improvement of the adsorption characteristics of stainless steel surfaces by sputter-deposited films. Metrologia 44, 161-166 (2007)

16. Naono, H., Hakuman, M.: Analysis of adsorption isotherms of water vapor for nonporous and porous adsorbents. J. Colloid Interface Sci. 145(2), 405-412 (1991)

17. He, M., Blum, A.S., Aston, D.E., Buenviaje, C., Overney, R.M.: Critical phenomena of water bridges in nanoasperity contacts. J. Chem. Phys. 114(3), 1355-1360 (2001)
18. Yaqoob, M.A., de Rooij, M.B., Schipper, D.J.: Design of a vacuum based test rig for measuring micro adhesion and friction force. High Performance Structures and Materials VI, pp. 261-274. (2012)

19. Fuller, K.N.G., Tabor, D.: The effect of surface roughness on the adhesion of elastic solids. Proc. R. Soc. Lond. A 345, 327-342 (1975)

20. Rabinovich, Y.I., Alder, J.J., Ata, A., Singh, R.K., Moudgil, B.M.: Adhesion between nanoscale rough surfaces II. Measurement and comparison with theory. J. Colloid Interface Sci. 232, $17-24(2000)$

21. Ata, A., Rabinovich, Y.I., Singh, R.K.: Role of surface roughness in capillary adhesion. J. Adhes. Sci. Technol. 16(4), 337-346 (2002) 